\title{
A MESOSCOPIC DRYING MODEL APPLIED TO THE GROWTH RINGS OF SOFTWOOD: MESH GENERATION AND SIMULATION RESULTS
}

\author{
Patrick Perré ${ }^{1}$ and Ian W. Turner ${ }^{2}$
}

\begin{abstract}
A mesoscopic drying model that enables the drying simulation of quartersawn and flatsawn wood sections consisting of several growth rings is presented. The procedure to generate the virtual board description directly from real sample images is also described. This virtual structure accommodates the prominent sample features, including its geometrical and physical properties, together with the density and structural variation across the growth rings. We give a synopsis of the sophisticated techniques developed specifically to generate this virtual description and exhibit the final computational meshes produced by the software for quartersawn and flatsawn sections. Low temperature drying simulations are then performed for both heterogenous and homogeneous model variants using these virtual descriptions and comparisons are made of the resulting MC field evolution. A highlight of these comparisons is that the heterogeneous model captures realistic drying effects, including the fast drying of earlywood and the late removal of liquid water in latewood. In comparing the drying of quartersawn and flatsawn boards we conclude that the effect of the heterogeneous nature of the MC fields is diminished somewhat when considering the flatsawn section over the quartersawn section.
\end{abstract}

Keywords: heterogeneous porous medium, wood properties, heat and mass transfer, computational modelling.

\section{INTRODUCTION}

Nowadays, the comprehensive set of equations governing heat and mass transport phenomena at the macroscopic level of a porous medium is well known and has been widely used to simulate several configurations, particularly the drying of materials such as wood (Perré and Turner 1999a-b, Perré et al. 2007). However, this macroscopic description has some drawbacks: its success hinges upon the correct physical and mechanical characterization of the medium, and under certain drying conditions the macroscopic approach can fail (Perré 2007). These drawbacks are undoubtedly the main motivation for developing multiscale approaches (Showalter 1993, Hornung 1997, Ray et al. 1997, Krabbenhoft and Damkilde 2004, Perré 2007). Different strategies, and hence possibilities, can be applied under these circumstances. In the case of time scale separation, the coupling between scales is sequential and the multiscale approach reduces to a change of scale. When the time scales overlap, a concurrent coupling has to be treated, which produces a real multiscale configuration that is more demanding in terms of computational resources and applied mathematical theory.

The correct characterisation of a porous medium is tedious, but nonetheless extremely important for its accurate computational modelling. New methods proposed for this task have appeared in the literature to predict the properties of the medium directly from the phase properties and the phase mor-

\footnotetext{
${ }^{1}$ AgroParisTech, INRA, ENGREF, 14, rue Girardet, F-54042, Nancy, France.

${ }^{2}$ School of Mathematical Sciences, QUT, Brisbane, Australia.

Corresponding author: perre@nancy-engref.inra.fr

Received: 30.07 2008. Accepted : 18.10. 2008.
} 
phology at the micro-scale (Adler 1992). Homogenisation, with its solid mathematical background, is among the most developed methods for predicting the macroscopic properties from the microscopic features of the medium (Hornung 1997, Bornert et al. 2001). For this method it is assumed that the micro- and macro-scales are independent. Unfortunately, in specific situations, such as mass migration in fibrous materials, this assumption may be breached and the homogenisation approach can fail (Chittaranjan 1997, Ludwig 1999). Under these circumstances the microscopic features can induce macroscopic behaviour and a multiscale formulation is necessary. In this instance, the information at the micro-scale must be continuously updated during the simulation of the macroscopic process, which leads to the notion of a double-porosity model. Note however, that in general, not all phenomena have to be treated in this manner.

In the field of multiscale modeling, the mesoscopic approach simply consists of accounting for the rapid variations of the material properties within the unit cell (Hornung 1997). This strategy requires the complex geometrical structure of the heterogeneous medium to be meshed and the grid to be refined enough for the small structure to be adequately captured. Such a strategy typically requires a substantial amount of computational resources unless the size of the unit cell is chosen just slightly smaller than the size of the domain $\Omega$. This strategy is adopted in this paper to model the drying of a piece of softwood comprised of several growth rings. In this case, the mesoscopic approach is a suitable way to account for the dramatic density variations in the wood sample across the growth rings between earlywood and latewood (typically from 280 to $1000 \mathrm{~kg} / \mathrm{m}^{3}$ ). Furthermore, the fact that all of the physical properties depend strongly on the density implies that the underlying material structure influences the simulation results significantly.

The paper is organized as follows. In the next section the mesoscopic model formulation is presented and then a brief review of our previous work (Perré and Turner 2001a-b, 2002) is given, together with a summary of the wood physical properties as functions of the material density. The techniques used to generate the virtual board description, including the underlying computational mesh, are outlined in the following section. Next, the simulation results for the low temperature drying of quartersawn and flatsawn sections of wood are exhibited for both the homogeneous and heterogeneous models and finally, in the last section of the paper, the conclusions of the work are summarized.

\section{THE MESOSCOPIC MODEL}

The conventional set of macroscopic equations that govern heat and mass transport in a homogeneous porous medium is well documented in the literature (Whitaker 1977, 1998). Modification of this set of equations to account for bound water has enabled this formulation to be used with great success to model the drying of a homogeneous sample of wood (consisting of either all sapwood or all heartwood) for several different drying configurations (Perré and Turner 1999a-b, Perré et al. 2007).

In the so-called mesoscopic model, these equations have been extended to capture material heterogeneity through the apparent density of the porous medium $\rho_{0}(\mathbf{x})$ and via the density variation of the material properties, capillary pressure, permeability, diffusivity and conductivity (Perré and Turner 2002). The resulting mathematical model is summarised briefly as follows:

\section{Liquid Conservation}

$$
\frac{\partial}{\partial t}\left(\rho_{o}(\mathbf{x}) \mathrm{X}+\varepsilon_{g} \rho_{v}\right)+\nabla \cdot\left(\rho_{w} \overline{\mathbf{v}}_{w}+\rho_{v} \overline{\mathbf{v}}_{g}+\overline{\rho_{b} \mathbf{v}_{b}}\right)=\nabla \cdot\left(\rho_{g} \overline{\overline{\mathbf{D}}}_{e f f} \nabla \omega_{v}\right)
$$


Air Conservation

$$
\frac{\partial}{\partial t}\left(\bar{\rho}_{a}\right)+\nabla \cdot\left(\rho_{a} \overline{\mathbf{v}}_{g}\right)=\nabla \cdot\left(\rho_{g} \overline{\overline{\mathbf{D}}}_{e f f} \nabla \omega_{a}\right)
$$

\section{Energy Conservation}

$$
\begin{gathered}
\frac{\partial}{\partial t}\left(\rho_{o}(\mathbf{x})\left(\mathrm{X} h_{w}+h_{s}\right)+\varepsilon_{g}\left(\rho_{v} h_{v}+\rho_{a} h_{a}\right)-\int_{0}^{\bar{\rho}_{b}} \Delta h_{w} d \rho-\varepsilon_{g} P_{g}\right) \\
+\nabla \cdot\left(\rho_{w} h_{w} \overline{\mathbf{v}}_{w}+\left(\rho_{v} h_{v}+\rho_{a} h_{a}\right) \overline{\mathbf{v}}_{g}+h_{b} \overline{\rho_{b} \mathbf{v}_{b}}\right) \\
=\nabla \cdot\left(\rho_{g} \overline{\overline{\mathbf{D}}}_{e f f}\left(h_{v} \nabla \omega_{v}+h_{a} \nabla \omega_{a}\right)+\overline{\overline{\mathbf{K}}}_{e f f} \nabla T\right)
\end{gathered}
$$

The gas and liquid phase velocities are given by the Generalised Darcy's Law:

$$
\overline{\mathbf{v}}_{\ell}=-\frac{\overline{\overline{\mathbf{K}}} \overline{\mathbf{k}}_{\ell}}{\mu_{\ell}} \nabla \varphi_{\ell}, \nabla \varphi_{\ell}=\nabla P_{\ell}-\rho_{\ell} g \nabla \chi \quad \text { where } \ell=w, g .
$$

The quantities $\varphi$ are known as the phase potentials and $\chi$ is the depth scalar. All other symbols have their usual meaning. The bound liquid flux is assumed proportional to a gradient in the bound water moisture content:

$$
\overline{\rho_{b} \mathbf{v}_{b}}=-\rho_{0}(\mathbf{x}) \overline{\overline{\mathbf{D}}}_{b} \nabla X_{b} .
$$

\section{Closure Conditions}

Because wood is a highly hygroscopic porous medium, bound liquid must be separated from free water in the definition of moisture content:

$$
X=X_{w}+X_{b} \equiv \frac{\varepsilon_{w} \rho_{w}}{\rho_{o}}+\min \left(X, X_{F S P}\right), \quad X_{F S P}=0.325-0.001 T
$$

The volume fractions of the liquid and gaseous phases are defined, in the usual sense, as. $\varepsilon_{w}=\phi S_{w}, \varepsilon_{g}=\phi\left(1-S_{w}\right), \varepsilon_{w}+\varepsilon_{g}=\phi$. The intrinsic phase air density is defined as $\bar{\rho}_{a}=\varepsilon_{g} \rho_{a}$ and the gaseous phase is a binary mixture of air and vapour, which is assumed to behave like an 1deal gas. During drying both the liquid and gaseous phases are evident within the porous medium. As a result of the curvature of the interface that exists between the liquid and the gas phases within the pores of the medium, the liquid pressure is less than the gas pressure and the capillary pressure represents that difference:

$$
P_{w}=P_{g}-P_{c} .
$$

The mass fractions of the air and vapour phases are denoted by $\omega_{a}$ and $\omega_{V}$ respectively. 


\section{Boundary and Initial conditions}

Initially the porous medium has some prescribed uniform temperature distribution and the pressure is constant throughout at the atmospheric value. The initial moisture content field must be determined by solving a nonlinear system that involves an equilibration of capillary forces throughout the medium, together with the requirement that an overall mass balance equation be satisfied (Perré and Turner 2002).

\section{Material Properties}

Recent research saw the determination of the material property variations across a growth ring of softwood (Perré and Turner 2001a-b). In that work, experimental observation and mathematical tools including image analysis, homogenization and a simple tracheid model enabled a complete set of correlations for capillary pressure $P_{c}\left(\rho_{0}, X_{w}, T\right)$, bound liquid diffusivity $\overline{\mathbf{D}}_{b}\left(\rho_{0}, X_{b}, T\right)$, thermal conductivity $\overline{\overline{\mathbf{K}}}_{\text {eff }}\left(\rho_{0}, X, T\right)$ and absolute permeability $\overline{\mathbf{k}}_{w}\left(\rho_{0}\right), \overline{\overline{\mathbf{k}}}_{g}\left(\rho_{0}\right)$ to be postulated. These properties have been used for the simulation results discussed throughout this text and the interested reader is referred to the reference papers for further details on the exact form of the correlations.

\section{Computation of the Wood Density at a Node}

Referring to Figure 2, we note that each control volume shares sub-control volumes that belong to different triangular finite elements. Each of these finite elements has associated with it an angle and a density depending on its location within the virtual board mesh. The apparent density needs to be determined at a node so that it can be used for the finite volume discretisation. We have used the area weighted averaging technique given in (Perré and Turner 2002), which is based on the following formula:

$$
\rho_{o}\left(\mathbf{x}_{p}\right)=\frac{\sum_{j=N b_{p}} A_{S C V_{j}}^{(e)} \rho_{o j}^{(e)}}{\sum_{j \in N b_{p}} A_{S C V_{j}}^{(e)}},
$$

where $\mathbf{x}_{p}$ represents the position vector of the node at the vertex-centre of the control volume.

The quantities $\sum_{j \in N b_{p}} A_{S C V_{j}}^{(e)} \rho_{o j}^{(e)} \quad$ and $\sum_{j \in N b_{p}} A_{S C V_{j}}^{(e)}$ represent the solid mass and the total area of the control volume respectively. The porosity $\phi^{\prime}\left(\mathbf{x}_{p}\right)$ can be computed for each control volume once the apparent density from (8) is known by using the equation:

$$
\phi\left(\mathbf{x}_{p}\right)=1-\frac{\rho_{o}\left(\mathbf{x}_{p}\right)}{\rho_{s}}, \rho_{s}=1530 \mathrm{~kg} \mathrm{~m} \mathrm{~m}^{-3}
$$

where $\rho_{s}$ is the density of the cell wall substance.

\section{Determination of the Initial Moisture Content Distribution}

The initial moisture content field must be computed prior to the commencement of the drying simulations. The average moisture content of the wood sample is initially supplied as an input to the code and this value is used to determine the initial distribution across the wood section. An important correlation needed for determining the initial moisture content field is the capillary pressure relation, which varies with liquid saturation, temperature and density. Typically, the pores in the latewood component of the annual ring are smaller than in the earlywood component. Consequently, stronger capillary forces become evident in latewood. The particular mathematical expression used in this work was postulated from image analysis performed on different locations within the annual ring of spruce (Perré 1997). 
By noting further that all capillary forces must be in equilibrium when the medium is in its initial state, the capillary pressures at each node of the computational mesh must be equal. If we let $P_{c o}$ be this equilibrium capillary pressure, then the following equation must hold for each control volume:

$$
P_{c_{P}}\left(S_{w}, T, \rho_{o}\right)=P_{c o}, \forall p=1,2, \ldots, N
$$

The moisture content for the control volume is given by

$$
X_{p}=\frac{\phi\left(\mathbf{x}_{p}\right) S_{p} \rho_{w}}{\rho_{o}\left(\mathbf{x}_{p}\right)}+X_{F S P} .
$$

Denoting the average moisture content by $\bar{X}$, then the equation that allows closure of this nonlinear system is given by:

$$
\frac{\sum_{p=1}^{N} A_{p} \rho_{o}\left(\mathbf{x}_{p}\right) X_{p}}{\sum_{p=1}^{N} A_{p} \rho_{o}\left(\mathbf{x}_{p}\right)}=\bar{X}, \quad A_{p}=\sum_{j \in N b_{p}} A_{S C V_{j}} .
$$

The above nonlinear system of $N+1$ equations can be solved for the unknown initial saturations $S_{w p}, p=1,2, \ldots, N$ at each node point and the equilibrium capillary pressure $P_{c o}$ using the Newton method (Perré and Turner 2002). Once the initial saturation values are known at all node points, it is then possible to deduce the initial moisture field. Figures 6 and 7 depict the initial moisture distribution computed for the quartersawn and flatsawn virtual boards. It is clear from these figures that the moisture is highest for the low-density regions (earlywood) and lowest for the high-density regions (latewood).

\section{Material properties as a function of density}

An entire work was proposed some years ago to obtain the variations of the transport properties within an annual ring of softwood (Perré and Turner 2001a-b). In that work several tools including image analysis, the virtual tracheid, fluid mechanics, homogenization, were used to propose expressions that define these properties as functions of the local density. Only the final expressions are collected hereafter. The interested reader is invited to refer to the above references for further explanation of the underlying theoretical considerations.

\section{Basic variable definitions}

$$
\begin{aligned}
\phi & \equiv \text { Porosity } \\
\rho_{s} & \equiv \text { Density of the cell-wall substance }\left(\rho_{\mathrm{s}}\right. \\
\rho_{0} & \equiv \text { Specific wood density (oven dry mas: } \\
\rho_{l} & \left.\equiv \text { Water density }\left(\rho_{l}=1000 \mathrm{~kg} \mathrm{~m}\right)^{-3}\right) \\
X & \equiv \text { Moisture content }(\mathrm{kg} \text { water } / \mathrm{kg} \text { of dry } \\
X_{l} & \equiv \text { Free water moisture content } \\
X_{b} & \equiv \text { Bound water moisture content } \\
X_{F F P} & \equiv \text { Fibre Saturation Point } \\
T & \equiv \text { Temperature }{ }^{\circ} \mathrm{C}
\end{aligned}
$$


The saturation variable is calculated according to the free water content only:

$$
S_{l}=\frac{X_{l}}{X_{l \max }} .
$$

Assuming that the volume of the pores is constant and that the density of bound water and free water are equal, the following expression has been derived for the volume fraction of the solid phase:

$$
\varepsilon_{s}=\frac{1+\frac{\rho_{s} X_{b}}{\rho_{l}}}{\left(\frac{\rho_{s}}{\rho_{0}}+\frac{\rho_{s}}{\rho_{l}}\left(X_{b}-X_{f s p}\right)\right)} .
$$

The porosity is defined as $\phi=\left(1-\varepsilon_{s}\right)$ and the volume fractions of the liquid and gaseous phases are given respectively by

$$
\varepsilon_{l}=\phi S_{l} \text { and } \varepsilon_{g}=\phi\left(1-S_{l}\right)
$$

\section{Capillary pressure}

The following mathematical expression has been elaborated from the averaged pore size partition determined by image processing for the same local density on several annual rings:

$$
\begin{aligned}
P_{c}\left(S_{l}, T, \rho\right) & =\sigma(T) \times\left\{\frac{3150}{S_{l}+1 \times 10^{-4}}-\frac{1047+3.368 \rho_{0}}{1.02-S_{l}}\right. \\
+ & \left.149.8 \rho_{0}\left(1-S_{l}\right)+52350+168.4 \rho_{0}-\frac{3150}{1+1 \times 10^{-4}}\right\}
\end{aligned}
$$

This expression assumes that the liquid partition minimizes the energy of the surface tension. In equation (16) $P_{c}$ is in Pascal $(\mathrm{Pa})$ and $\sigma(T)=(77.5-0.185 T) \times 10^{-3} \mathrm{Nm}^{-1}$ represents the surface tension of water. When moving from low to high-density values, the capillary pressure increases in magnitude, due to the reduction in averaged pore diameter and the saturated moisture content decreases.

\section{Tracheid model}

The average tracheid shape evolves significantly between earlywood and latewood. In order to account for this variation, a virtual tracheid model has been developed from anatomical observations. Recall that the tracheids are created in the radial direction by the division of the same initial cell in the cambium, the tangential dimension $\left(a_{T}\right)$ is almost constant. The value $a_{T}=50 \theta \mu \mathrm{m}$ was assumed here. Accounting for tracheid overlapping, the length in the longitudinal direction was taken to be $a_{L}=1.5 \mathrm{~mm}$.

In order to be consistent with the observation, the radial dimension and the cell wall thickness $t$ of this virtual tracheid were assumed to depend on the local wood density as follows:

$$
\begin{aligned}
a_{R}(\rho)= & 0.575 \times 10^{-4}-0.375 \times 10^{-7} \rho \quad(\mathrm{m}), \\
t_{v}(\rho)= & .53733443 \times 10^{-6}+ \\
+ & .66577831 \times 10^{-8} \rho+.10275104 \times 10^{-10} \rho^{2} \\
- & .82567033 \times 10^{-14} \rho^{3} \quad(\mathrm{~m}) .
\end{aligned}
$$




\section{Liquid permeability}

Accounting for the viscous resistance in the tracheid lumen and in the pits, the following correlations were derived for the liquid permeability in the radial, tangential and longitudinal directions:

$$
\begin{aligned}
& k_{R}(\rho)=\frac{n_{R}(\rho) a_{R}(\rho)}{2 a_{T} a_{L} \alpha(\rho)}, \\
& k_{T}(\rho)=\frac{n_{T}(\rho) a_{T}}{2 a_{R}(\rho) a_{L} \alpha(\rho)}, \\
& k_{L}(\rho)=\frac{a_{L}}{a_{T} a_{R}(\rho)\left(\frac{\alpha(\rho)}{n_{L}(\rho)}+\frac{a_{L}}{\beta(\rho)}\right)} .
\end{aligned}
$$

Here, $\alpha(\rho)$ represents the resistance to fluid flow through one pit, and $n_{L}, n_{R}, n_{T}$ are the number of available pits in each flow direction. $\beta(\rho)$ is the flow resistance within the tracheid lumen.

$$
\begin{aligned}
& \alpha(\rho)=c_{3}\left(t_{v}(\rho)+c_{4}\right) \\
& \beta(\rho)=0.27514032 \times 10^{-18}-0.71386999 \times 10^{-21} \rho+0.46368212 \times 10^{-24} \rho^{2} \\
& +0.12115020 \times 10^{-27} \rho^{3}-0.14614421 \times 10^{-30} \rho^{4} \\
& n_{R}=\gamma\left(a_{T}-2 t_{v}(\rho)\right), n_{T}=\gamma\left(a_{R}-2 t_{v}(\rho)\right), n_{L}=n_{R}+n_{T} \text {, }
\end{aligned}
$$

The value $\gamma=\frac{25}{a_{T}}$ appears to give a reasonable total number of pits equal to a 50 per overlapping zone in earlywood. The coefficients and are chosen to calibrate the permeability relations to lie within the range of experimental measurement $\left(c_{3}=1.8 \times 10^{24}\right.$ and $\left.c_{4}=2 \times 10^{-6}\right)$. With these values, the difference between earlywood and latewood is approximately a ratio of ten, which is in agreement with the values proposed by Bolton and Petty (1978).

\section{Gas permeability}

The primary contrast between the gaseous and liquid phase permeabilities concerns the aspiration of the pits. An "aspiration" function that depends on the wall thickness was introduced. The functional form of the aspiration $A_{s}\left(t_{v}\right)$ has been assumed to follow an exponential shape that results in 5\% of non-aspirated pits in earlywood up to $100 \%$ in latewood. This function simply changes the number of available pits in the radial and transverse directions as follows:

$$
n_{R}=A_{s}\left(t_{v}\right) \gamma\left(a_{T}-2 t_{v}(\rho)\right), n_{T}=A_{s}\left(t_{v}\right) \gamma\left(a_{R}-2 t_{v}(\rho)\right) \text {. }
$$




\section{Bound liquid diffusivity}

In this section, the gradient of bound water is assumed to be the driving force. This allows two pseudo-diffusivities to be defined (bound water in the cell wall and air in the pores):

$$
\begin{aligned}
& \hat{D}_{b}=\rho_{s} D_{b}, \\
& \hat{D}_{v}=\frac{M_{v}}{R T} D_{v} \frac{\partial P_{v}}{\partial X_{b}}
\end{aligned}
$$

where $\hat{D}_{b}=\rho_{s} \exp \left(-12.8183993+10.8951601 X_{b}-\frac{4300}{T+273.15}\right)($ Stamm 1959).

Using homogenization results, the followings correlations were proposed:

$$
\begin{aligned}
& \tilde{D}_{b}^{R}=A_{\text {series }}\left(1+1.6\left(\varepsilon_{g}\right)^{1.8}\right), \\
& \tilde{D}_{b}^{T}=1.8 \times A_{\text {series }},
\end{aligned}
$$

$$
\text { with } \quad A_{\text {series }}=\frac{1}{\frac{\varepsilon_{s}}{\hat{D}_{b}}+\frac{\varepsilon_{g}}{\hat{D}_{v}}} \text {. }
$$

A small percentage of ray cells has to be put in parallel to the cell arrangement giving the final form of the radial macroscopic diffusivity as:

$$
\rho_{0} D_{b}^{R}=\left(1-\varepsilon_{\text {ray }}\right) \tilde{D}_{b}^{R}+\varepsilon_{\text {ray }} \times \tilde{D}_{\text {ray }}^{R}
$$

Typical values for $\varepsilon_{\text {ray }}$ and $\tilde{D}_{\text {ray }}^{R}$ are .05 and $0.1 \times \hat{D}_{v} 1$ respectively (values adapted from Siau 1995). In order to be consistent, the same amount of ray cells should be put in series with tracheids in the tangential direction:

$$
\rho_{0} D_{b}^{T}=\frac{1}{\frac{1-\varepsilon_{\text {ray }}}{\tilde{D}_{b}^{T}}+\frac{\varepsilon_{\text {ray }}}{\tilde{D}_{\text {ray }}^{T}}} .
$$

Due to the considerable length of wood fibers, the longitudinal macroscopic diffusivity is simply derived using a parallel model of the form:

$$
\rho_{0} D_{b}^{L}=\varepsilon_{s} \hat{D}_{b}+\varepsilon_{g} \hat{D}_{v} .
$$




\section{Thermal conductivity}

The conductivity was obtained using homogenization in the R-T plane and using a parallel flow model in the longitudinal direction:

$$
\begin{aligned}
& \tilde{\lambda}_{0}^{R}=0.46 \times \lambda_{\text {series }}+0.54 \times \lambda_{\text {parallel }}, \\
& \tilde{\lambda}_{0}^{T}=\left(\varepsilon_{g} \lambda_{\text {air }}^{n}+\varepsilon_{s} \hat{\lambda}_{s \perp}^{n}\right)^{\frac{1}{n}} \quad \text { with } n=0.6, \\
& \lambda_{0}^{L}=\varepsilon_{g} \lambda_{\text {air }}+\varepsilon_{s} \hat{\lambda}_{s / /} \text { with } \hat{\lambda}_{s / /} \approx 2 \times \hat{\lambda}_{s \perp},
\end{aligned}
$$

where

$$
\lambda_{\text {series }}=\frac{1}{\frac{\varepsilon_{s}}{\hat{\lambda}_{s \perp}}+\frac{\varepsilon_{g}}{\lambda_{\text {air }}}} \text { and } \lambda_{\text {parallel }}=\varepsilon_{s} \hat{\lambda}_{s \perp}+\varepsilon_{g} \lambda_{\text {air }} \text {. }
$$

Properties with a subscript of 0 denote thermal conductivities at a moisture content equal to zero. $\lambda_{\text {air }}=0.023 \mathrm{~W} \mathrm{~m}^{-1} \mathrm{~K}^{-1}$ and $\hat{\lambda}_{s \perp}=0.5 \mathrm{~W} \mathrm{~m}^{-1} \mathrm{~K}^{-1}$. The effect of ray cells must be taken into account

$$
\begin{aligned}
& \lambda_{0}^{R}=\left(1-\varepsilon_{\text {ray }}\right) \tilde{\lambda}_{0}^{R}+\varepsilon_{\text {ray }} \times \tilde{\lambda}_{\text {ray// }}, \\
& \lambda_{0}^{T}=\frac{1}{\frac{1-\varepsilon_{\text {ray }}}{\tilde{\lambda}_{0}^{T}}+\frac{\varepsilon_{\text {ray }}}{\tilde{\lambda}_{\text {ray } \perp}}} \text { with } n=0.6 .
\end{aligned}
$$

$\lambda_{\text {ray } \perp}$ and $\lambda_{\text {ray// }}$ are evaluated using equations (24) respectively with a density of $500 \mathrm{~kg} \cdot \mathrm{m}^{-3}$.

For any value of moisture content, the final expressions for conductivity read:

$$
\begin{aligned}
& \lambda^{T}(\rho, X)=\left(\left(1-S_{l}\right)\left(\lambda_{0}^{T}\right)^{n}+S_{l}\left(\phi \lambda_{l}^{n}+\varepsilon_{s} \hat{\lambda}_{s \perp}^{n}\right)\right)^{\frac{1}{1}}, \\
& \lambda^{R}(\rho, X)=\left(\left(1-S_{l}\right)\left(\lambda_{0}^{R}\right)^{n}+S_{l}\left(\phi \lambda_{l}^{n}+\varepsilon_{s} \hat{\lambda}_{s \perp}^{n}\right)\right)^{\frac{1}{1}}, \\
& \left.\lambda^{L}(\rho, X)=\left(1-S_{l}\right)\left(\tilde{\lambda}_{0}^{L}\right)^{\prime}+S_{l}\left(\phi \lambda_{l}+\varepsilon_{s} \lambda_{s / l}\right)^{n}\right)^{\frac{1}{1}},
\end{aligned}
$$

where $n=0.6$ for the three expressions. 


\section{Mesh generation}

It must be recognized that if realistic simulations of the drying of wood cross-sections is to be realized using our heat and mass transfer code, considerable effort must be injected into the development of the virtual board structure to account for the growth ring descriptions. This virtual board acts as a repository for all of the important features of the sample, including its geometrical and physical properties. In this work, the gap between the section images and the CV-FE mesh was filled using customized software developed some years ago (Perré 2005). This software, MeshPore, was primarily developed for wood at the macroscopic, anatomical and cellular levels, but can be extended to various kinds of materials and various scales of observation. MeshPore is entirely developed in FORTRAN 90. Using a graphical library (Winteracter) the compiled version is a Windows-like application, which operates by menus, dialog boxes and mouse control.

A specific data structure has been developed for use in MeshPore. Based on the concept of directed line segments (Gonzales and Woods 1992), this structure was conceived to fulfill several purposes: to handle any shape (including closed or open contours), to be able to change the number of nodes of each chain, to distinguish the contour structure and the location of geometrical points, and so on. The basic elements are points and nodes and a contour consists of a chain of nodes, with each of them pointing towards a geometrical point. Each point has geometrical information associated with it, while each node has connection information. A contour is defined by a chain, which consists of a set of connected nodes (as described in Figure 1). Each node of a chain has its previous node ( 0 for the first node) and its next node ( $=0$ for the last node). A node also points towards one geometrical point and note that several nodes can point towards the same point, for example, a chain is closed only if the first and the last node point towards the same point. This important feature allows topological properties of a set of chains to be kept even when a geometrical point is moved. The most important steps allowed by this application are listed below:

- $\quad$ image segmentation and description of contours by segment chains,

- $\quad$ node and point editing (add, delete, move, define as fixed...),

- $\quad$ chain editing (add, delete, close, join, split...),

- addition of a chain type for further control of boundary conditions,

- chain re-sampling and orientation for further control of the mesh properties, mesh generation (the file format is suitable for EasyMesh, developed by Bojan Niceno: http://www-dinma.univ.trieste. it/nirftc/research/easymesh),

- $\quad$ element editing (material type, local angle and density, spread angle and material type throughout connecting zones). 


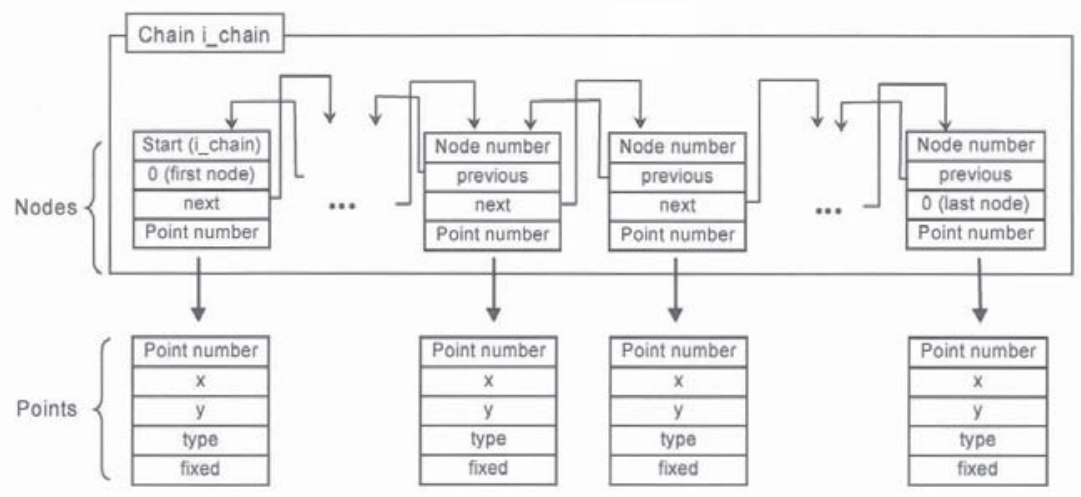

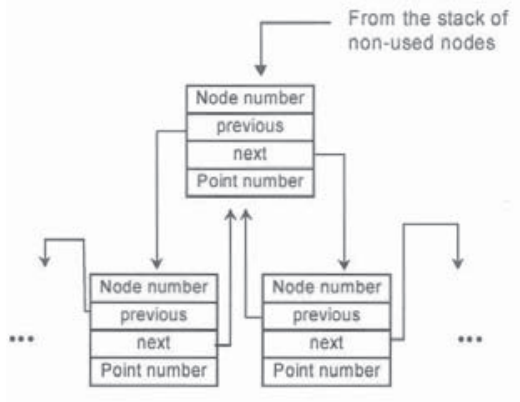

Adding a node

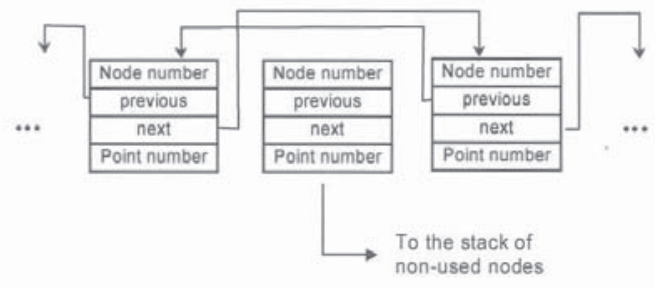

Removing an existing node.

Figure 1: Synopsis of the data structure used in MeshPore, which enables simple operations such as adding or removing a node from an existing chain.

The operator can choose any solution between a full automatic treatment (suitable only on excellent images) to a complete manual treatment. Usually, an automatic segmentation allows a suitable chain structure to be generated. Then, the operator can correct obvious problems due to image defaults. Once a proper set of chains is obtained, they can be changed easily through an automatic change function, for example, the chain and hence the mesh refinement, or the chain orientation to mesh, or the internal holes defined by closed chains.

The concept of a fixed point is very important for a suitable mesh generation. It allows the corner to be kept when changing the chain refinement and allows specific locations to remain fixed during the mesh generation. By this way, these specific points will be kept as triangle vertices after mesh generation by EasyMesh. 
In this work, heterogeneous sections of softwood have been prepared using MeshPore. The starting point is an image of a carefully polished Spruce section. The following operations are applied to this image (refer to Figure 2):

- Density calibration of the grey level. In this procedure an affine relationship is assumed between the density and the grey level $\mathrm{gl}(\rho=a \mathrm{x} g l+b)$. The average density and the classical density variation between earlywood and latewood is used to identify the two parameters.

- Automatic generation of the external contour.

- Manual generation of internal contours connected to this external contour to define the annual growth rings. Points of these contours are fixed points.

- Manual generation of internal contours in between successive annual growth rings. These contours inform the mesh generator to produce coarser elements in this region of more homogeneous density. Points of these contours are referred to as free.

- Mesh generation using EasyMesh.

- Generation of material angle, next to the internal contours that define the tangential direction, using MeshPore. The angle is then spread throughout the mesh using a subtle modulo- $180^{\circ}$ average routine, over two or three neighbors.

- Generation of the CVs used to solve the balance equations. The control volumes are formed directly from the triangular mesh by joining the centroids of the triangles to the midpoints of the triangle vertices. 
a)

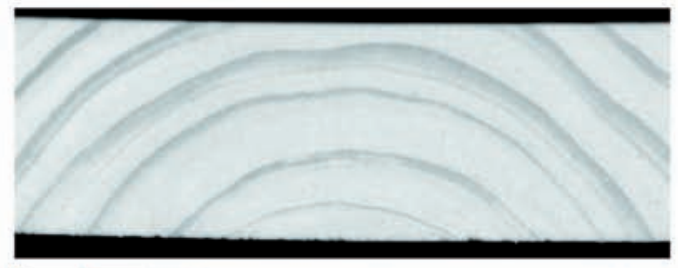

b)

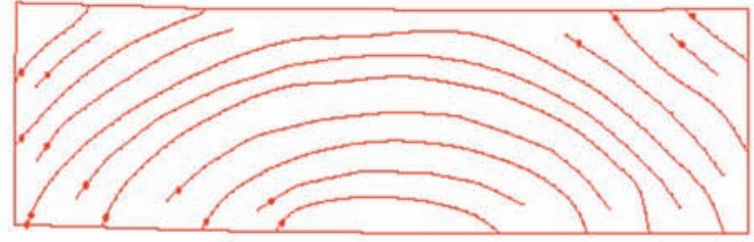

c)

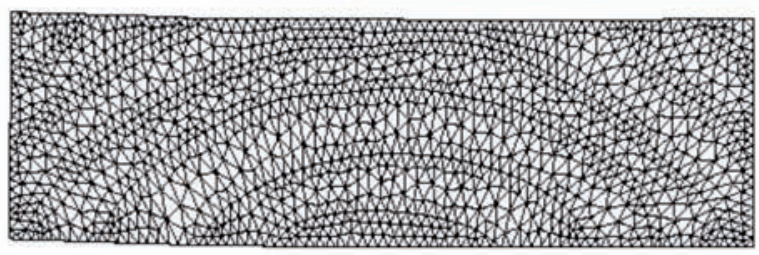

d)

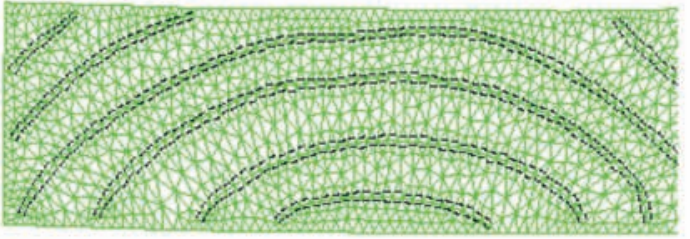

e)

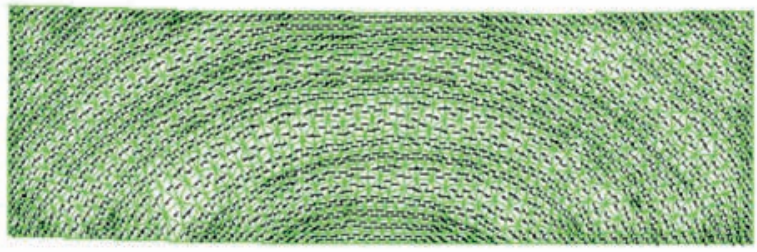

f)

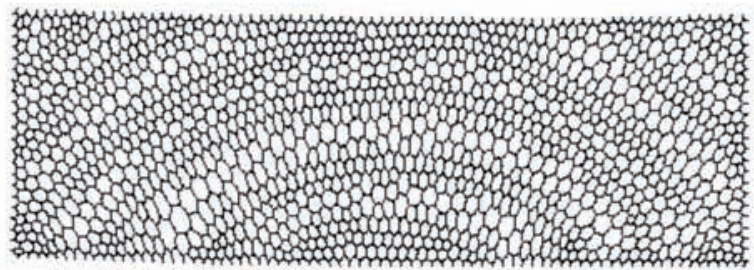

Figure 2: Meshing a spruce section at the macroscopic level: a) initial macroscopic view of the flatsawn section, b) manual contours of the annual growth rings and within the annual ring (control of the mesh refinement), c) mesh generated by easymesh d) material angle of all elements in contact with the annual ring contours e) material angle spread throughout the section $\mathrm{f}$ ) local density map with the control volumes constructed from the Femesh (2307 elements). 
The simulation results using both the homogeneous and heterogeneous drying model formulations are reported for the drying of the flatsawn and quartersawn sections using the mesh shown in Figure 2 and the refined mesh in Figure 3 respectively. For this R-T cross-sectional 2-D model, that prevents the highly permeable longitudinal direction to be taken into account, low temperature drying conditions with a dry bulb temperature of $60^{\circ} \mathrm{C}$ and wet bulb temperature of $45^{\circ} \mathrm{C}$ have been used. The air velocity used for the simulations was $2 \mathrm{~m} / \mathrm{s}$, yielding heat and mass exchange coefficients of $15 \mathrm{~W} \mathrm{~m}^{-2} \mathrm{~K}^{-1}$ and $0.015 \mathrm{~m} \mathrm{~s}^{-1}$ respectively. Attention will be focused here on the specific behavior that can be captured by the heterogeneous model in comparison to the homogeneous variant.

a)

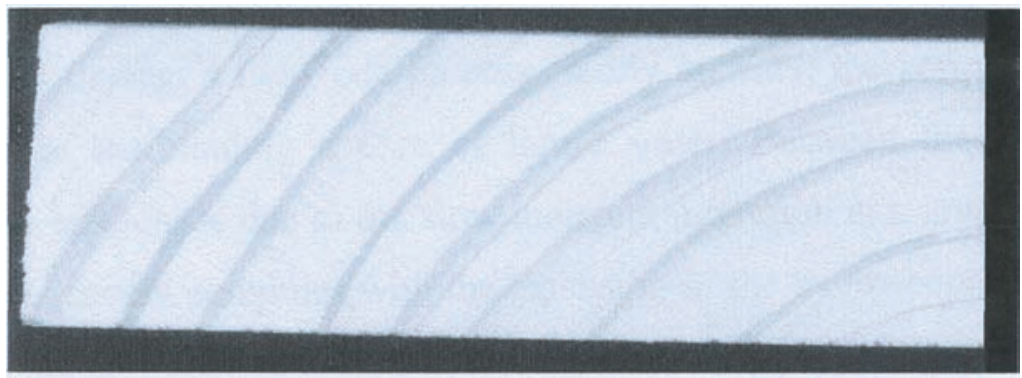

b)

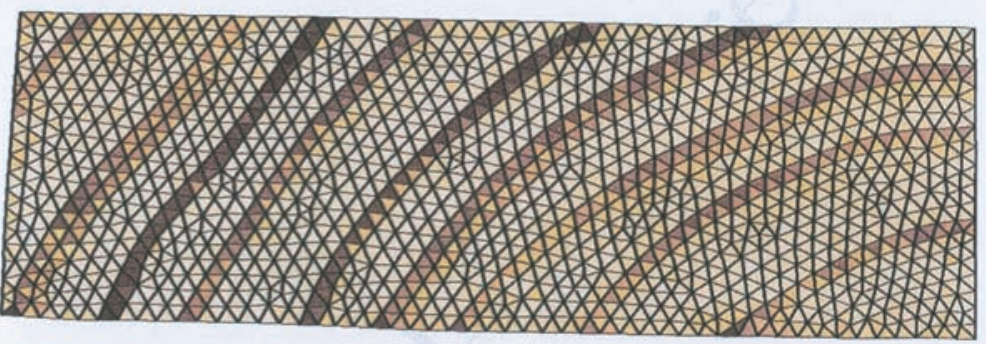

c)

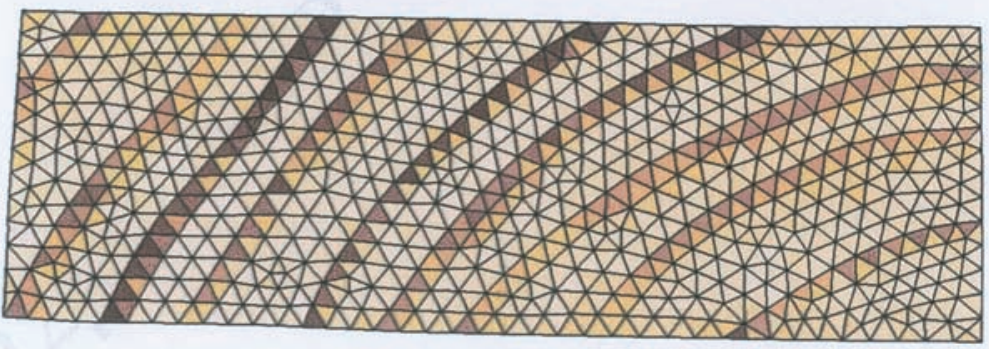

Figure 3: Possibilities offered by the mesh generation procedure: once the contours are constructed from the initial image using MeshPore, the mesh refinement can be easily chosen through the contour refinement.

Example for a quartersawn board: refined mesh $=2068$ elements, coarse mesh $=1163$ elements .

\section{The homogeneous model}

The first test was computed with the homogeneous quartersawn section. For this test, the physical properties do not depend on the position. They are calculated from the property values corresponding to the averaged density of this section $\left(441 \mathrm{~kg} / \mathrm{m}^{3}\right)$. The initial moisture content is uniform and equal to $170 \%$ (dry basis). Note however that the spatial variation of the grain angle used in this run is as calculated by the mesh generation procedure. 
It is clear from the three carpet plots depicted in Figure 4 that the moisture content variation in space for the homogeneous model is of a classical nature, with a smooth progression of the moisture content from its initial constant state to the equilibrium value established by the drying air characteristics. After 2 hours of drying, the moisture content starts to fall at the exchange surfaces, with a pronounced effect along the edges where two orthogonal exchange surfaces exist. After 10 hours of drying, a two-zone process clearly appears; the peripheral zone is within the hygroscopic range surrounding a core of liquid water. Between these two zones, the deep moisture content gradient is due to the slow moisture migration at a low content of liquid water. Then, the drying process continues with the extension of the hygroscopic zone and the reduction of the liquid domain, which continues to diminish until it eventually vanishes from the section core.
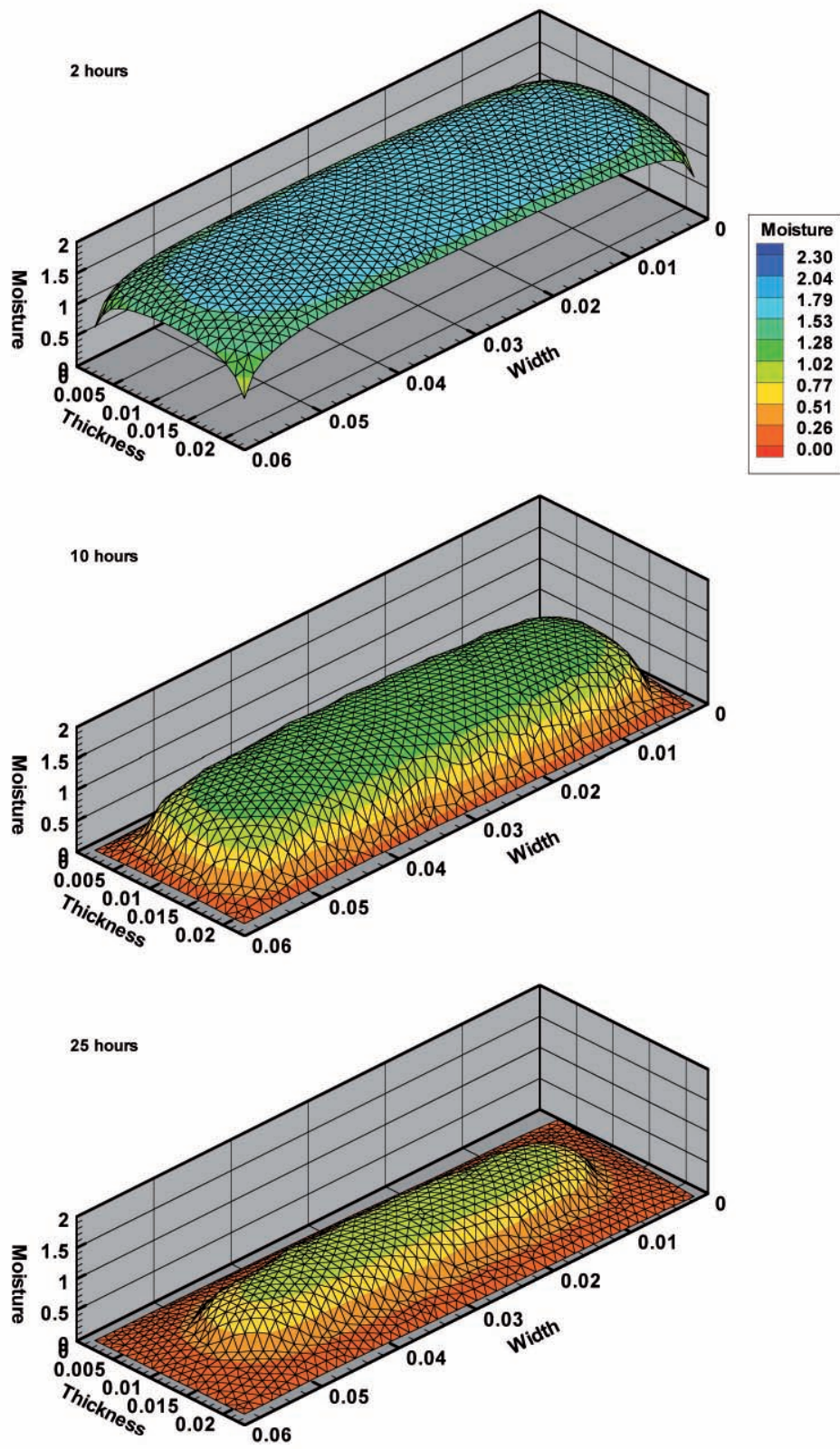

Figure 4: Simulated MC fields for the homogeneous quartersawn section (2, 10 and 25 hours of drying) 
The averaged moisture content decreases from 170\% down to about $25 \%$ in 40 hours (Figure 5). The difference in drying kinetics between the surface (mid-width) and the core of the section highlights the large moisture content gradient that exists throughout the process. Two distinct periods are evident on the temperature evolution, namely the constant rate period and the falling rate period. Surprisingly, the core temperature is larger than the surface temperature at the end of the constant drying rate period. This is due to a two-dimensional effect: the edges dry faster and are first to enter the second drying rate period. As a consequence, the temperature is larger than the wet bulb temperature, heating up the core of the section by conduction, while the surface at the mid-width remains at the wet bulb temperature as a result of the evaporation.

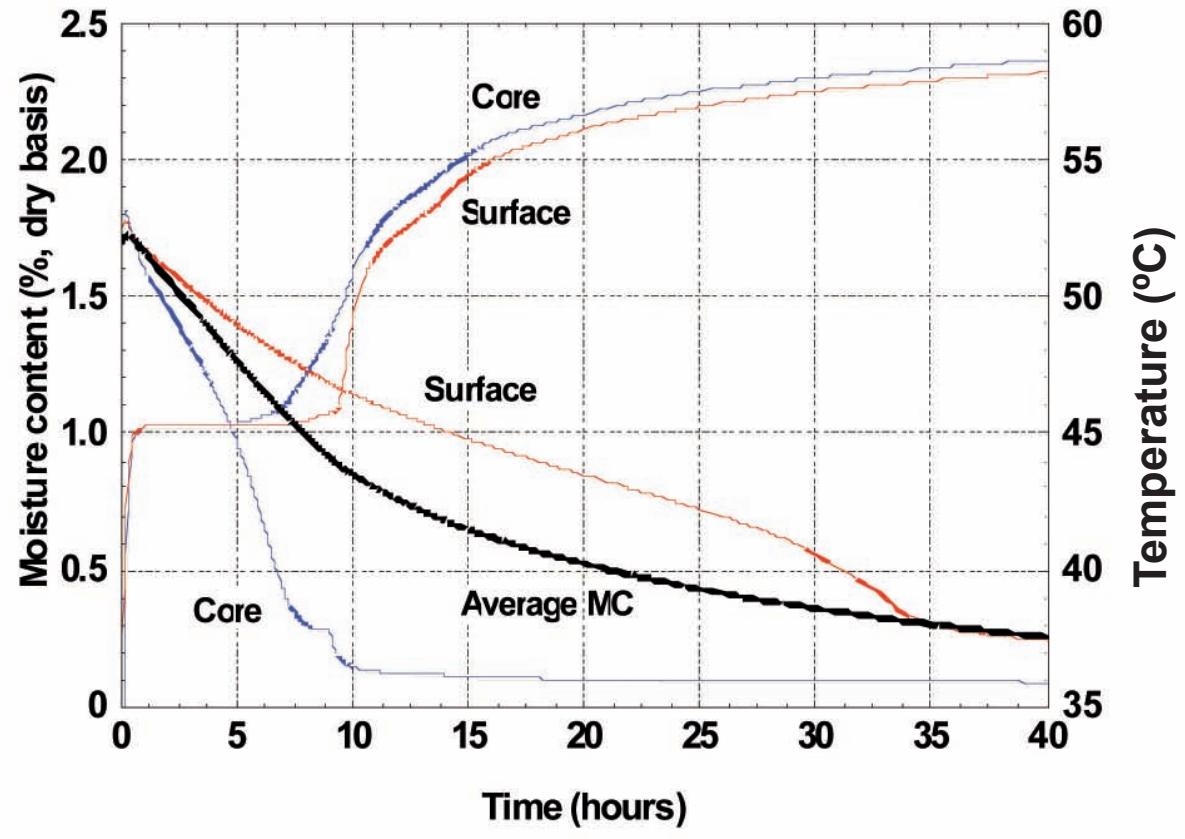

Figure 5: Drying kinetics obtained for the homogeneous quartersawn section: average moisture content and surface and core position (MC and temperature).

\section{The quartersawn heterogeneous section}

In the case of the heterogeneous model the initial moisture content field is not uniform and has to be computed prior to the commencement of the drying simulation. This process results in a moisture content field that ensures a uniform liquid pressure (Perré and Turner 2002). The influence of the strong density variation across the growth rings can be observed clearly, with the initial moisture field being higher in the earlywood regions and lower in the latewood regions of the wood sample (see Figure 6). After 4 hours of drying, a "macroscopic" moisture content gradient is evident (from the inner part of the section towards the exchange surfaces), together with a "microscopic" moisture gradient due to the local heterogeneities of the porous medium. This is a typical consequence of the mesoscopic approach. 

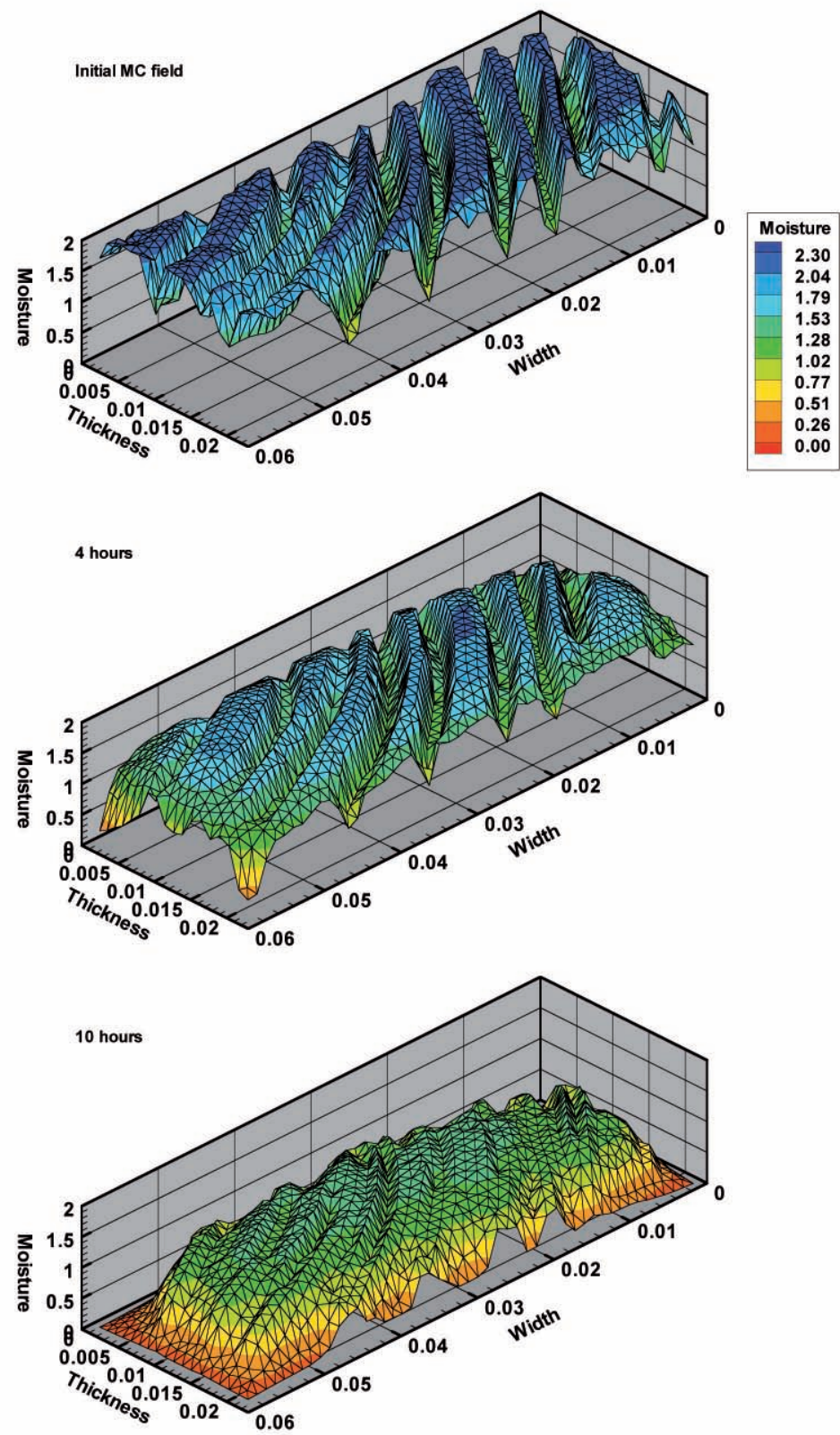

Figure 6: Simulated MC fields for the heterogeneous quartersawn section (initial field, 4 and 10 hours of drying).

Keeping in mind that the pores in the latewood component of the annual ring are smaller than in the earlywood component, stronger capillary forces become evident in latewood. As the drying proceeds, the earlywood locations are first to tend towards the fibre saturation point and thereafter the latewood locations attain this value. This leads to what can be coined as an "inversion" of the moisture content field whereby the MC of earlywood becomes less than the MC in latewood. This inversion starts to appear at 10 hours of drying and is fully developed by 20 hours (see Figure 7). Once the surface moisture content reaches the hygroscopic range a reduction in the external vapour flux causes the overall drying rate to slow. The drying air now begins to heat the board and the sample case appears dry. Two zones are now clearly evident - an inner zone where liquid migration again distinctly follows the 
growth ring pattern, and a surface zone where only bound water migration and water vapour diffusion occur. Then, as for the homogeneous model, the zone of liquid water reduces and finally disappears in the core of the section. Note that, in the domain of bound water, the liquid pressure is no longer involved and the field in the peripheral zones resembles that computed by the homogeneous model.

The drying kinetics computed for the heterogeneous sample shown in Figure 8 are slightly faster than those depicted for the homogeneous model given in Figure 5, with an averaged moisture content decreasing from $170 \%$ down to about $20 \%$ in around 40 hours. This figure succinctly highlights the inversion of the drying curve between earlywood and latewood, both at the surface and in the core of the section. Indeed, at a same "macroscopic" location, the latewood part starts to dry significantly only once the earlywood part next to it reaches a MC low enough for its capillary pressure to attain the one of saturated latewood.
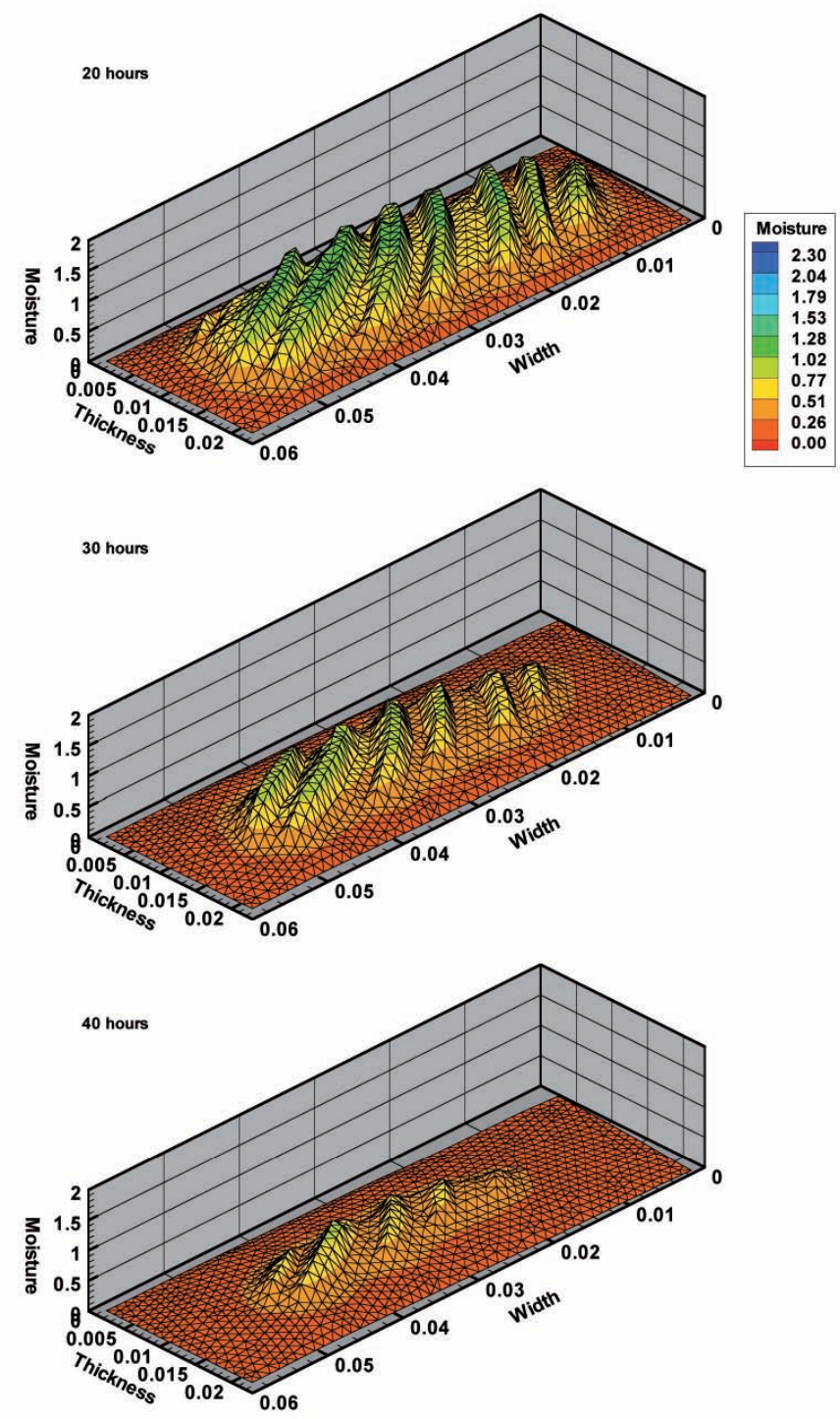

Figure 7: Simulated MC fields for the heterogeneous quartersawn section (20, 30 and 40 hours of drying). 


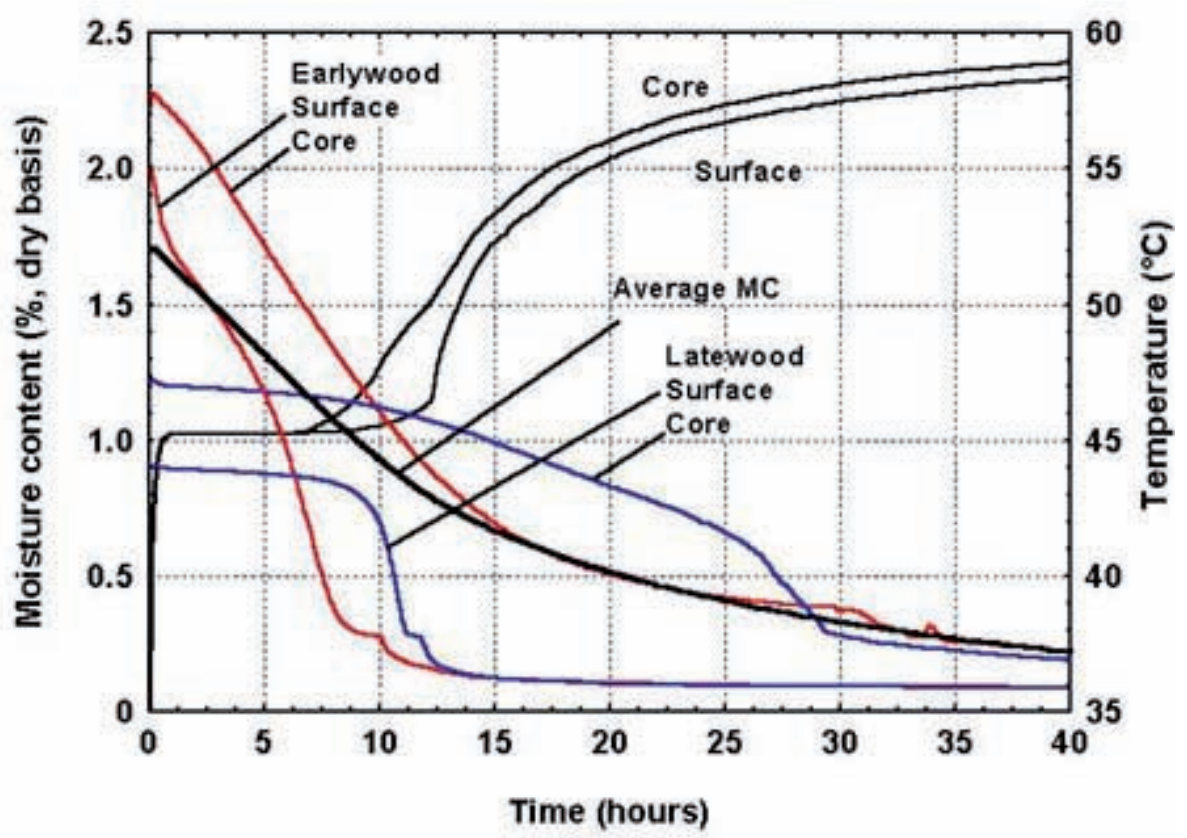

Figure 8: Drying kinetics obtained for the heterogeneous quartersawn section: average moisture content and typical earlywood and latewood points (surface and core).

Note finally that although there is a considerable impact on the moisture evolution due to the local heterogeneities in the board, this impact is almost unnoticeable in the temperature fields. For this reason, only a single carpet plot of the temperature distribution for the heterogeneous section has been exhibited in Figure 9. At 13 hours of drying, the edges of the section have already entered the falling rate period, which is evidenced by the significant increase in temperature along the edges to values $\left(\sim 55^{\circ} \mathrm{C}\right)$ that are higher than the wet bulb temperature. At the mid-width of the sample one can observe fluctuations in the temperature field, which can be easily explained by noting the evolution of the local moisture content in these regions. In particular, some latewood parts of the surface still contain liquid water that cause the temperature to remain at the wet bulb temperature, while other parts of the sample that have entered into the hygroscopic domain start to increase in temperature with the aid of the higher edge temperatures.

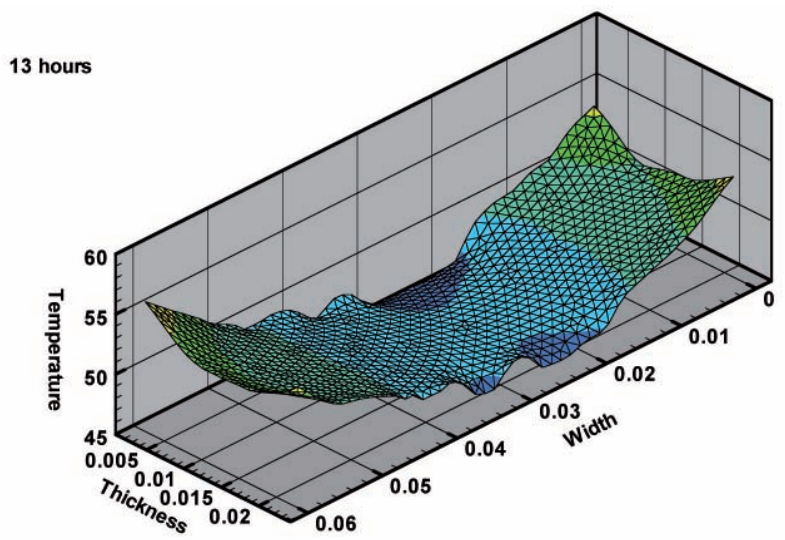

Figure 9: One selected temperature field for the heterogeneous quartersawn section at 13 hours of drying. 


\section{The false quarter-sawn board}

The results computed with the flatsawn section shown in Figure 10 depict several similarities to the quartersawn section, including the shape of the initial moisture content field, the contrast between the liquid and the hygroscopic zones, and the inversion of moisture content between earlywood and latewood. However, one major difference has to be emphasised: the heterogeneities due to the annual rings now act in series rather than in parallel. Therefore, the difference between the macroscopic and the mesoscopic effects is reduced, with a larger effect of the macroscopic behaviour evident. Consequently, the MC field at 15 hours resembles that of the homogeneous model. This trend can also be confirmed from the proceeding figures of the evolution of the MC fields, which are not shown here.
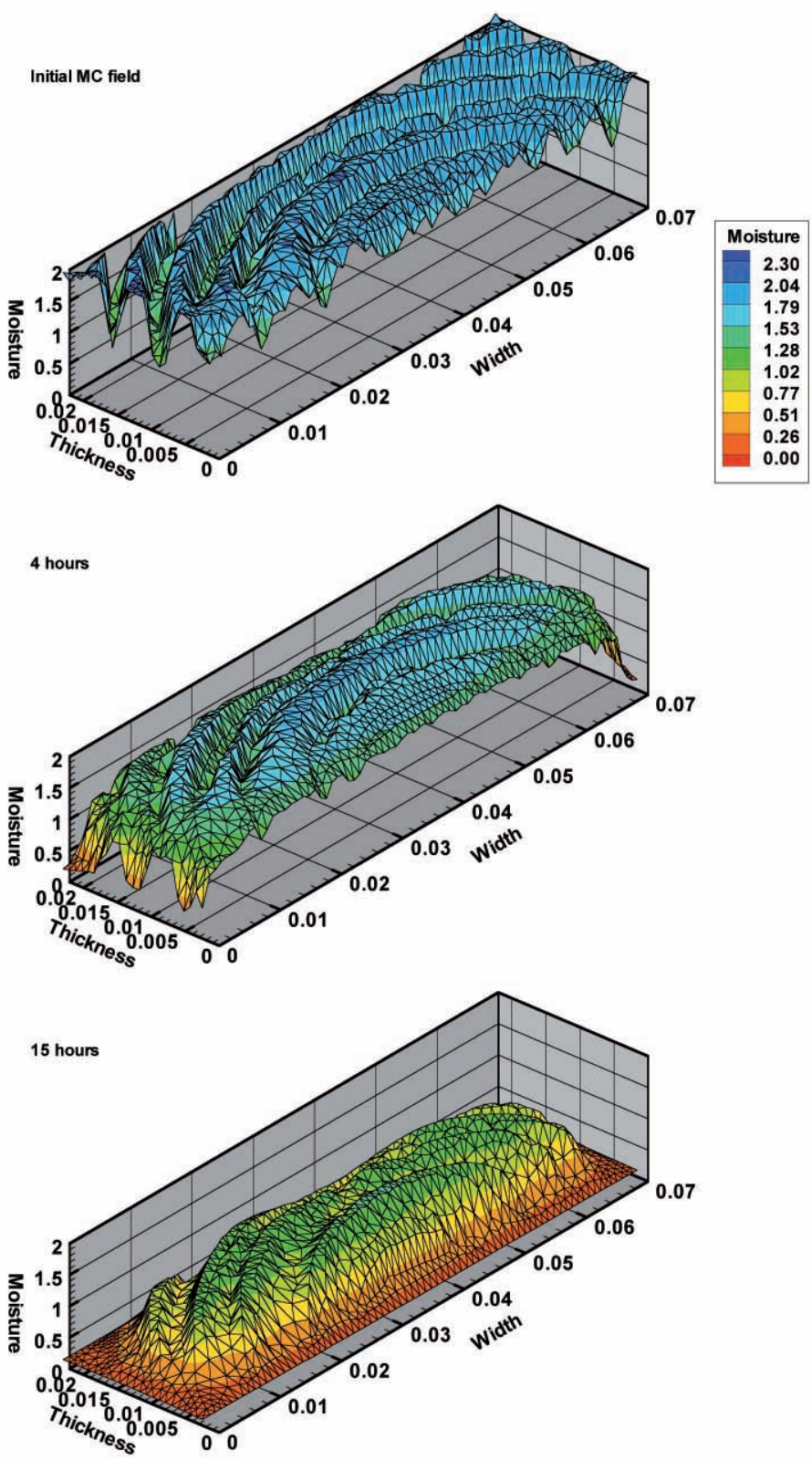

Figure 10: Simulated MC fields for the heterogeneous flatsawn section (initial field, 4 and 15 hours of drying). 


\section{CONCLUSIONS}

We have reviewed our previous research concerning a heterogeneous wood drying model and revisited some of the important aspects of the background theory. In particular, we have summarised the wood physical properties as functions of density for use in this model. Another essential requirement of the model is the underlying virtual board description that captures directly from wood section images the material structure and density variation across growth rings. We have described the sophisticated software that has been developed specifically to generate this virtual description for both quartersawn and flatsawn sections and exhibited the final meshes from which drying simulations can be performed.

Low temperature drying simulations were then carried out for both heterogenous and homogeneous model variants and comparisons were made of the resulting $\mathrm{MC}$ field evolution. It was clear that a substantially different behaviour was observed for the heterogeneous model, which enabled more realistic drying effects to be realised, including the heterogeneous initial MC field, the fast drying of earlywood, and the late removal of liquid water in latewood. Another key finding was that whilst dramatic changes were observed for the MC fields, little impact could be noted in the associated temperature distributions for the sample. Nevertheless the heterogeneous model was able to reveal some subtleties along the edges that are not possible to capture using the homogeneous model. In comparing the drying of quartersawn and flatsawn boards we remark that the effect of the heterogeneous nature of the MC fields is diminished somewhat when the growth rings act in series (flatsawn) rather than in parallel (quartersawn).

The next stages of the research are :

- to validate the simulated results using non-destructive techniques allowing the moisture content fields to be obtained with a relevant spatial resolution. By its ability to focus on water only, NMR imaging (Deurer et al. 2002, Almeida et al. 2008) is certainly more adapted for this purpose than X-ray tomography (Wiberg and Morén 1999).

- to extend the current formulation to a completely three-dimensional setting that accounts for the longitudinal effects evident during drying, which are extremely important when simulating high temperature drying or vacuum drying processes.

\section{REFERENCES}

Adler, P.M. 1992. Porous Media: Geometry and Transports. Butterworth-Heinemann Series in Chemical Engineering.

Almeida, G.; Leclerc, S.; Perré, P. 2008. NMR imaging of fluid pathways during drainage of softwood in a pressure membrane chamber. J. of Multiphase Flow 34: 312-321.

Bornert, M. ; Bretheau, T. ; Gilormini, P. 2001. Homogénéisation en mécanique des matériau. Vol. 1 - 2, Hermes Science, Paris.

Bolton, A. J.; Petty, J. A.. 1978. A model describing axial flow of liquids through conifer wood. Wood Science and Technology 12: 37-48.

Chittaranjan, R.; Ellsworth, T.R.; Valocchi, A.J.; Boast, C.W. 1997. An improved dual porosity model for chemical transport in macroporous soils. Journal of Hydrology 193: 270-292.

Deurer, M.; Vogeler, I.; Khrapitchev, A.; Scotter, D. 2002. Imaging of water flow in porous me- 
dia by magnetic resonance imaging microscopy. J. Environ. Qual. 31: 487-493.

Gonzales, R.C.; Woods, R.E. 1992. Digital image processing. Addison-Wesley, Reading, Massachusetts.

Hornug, U. 1997. Homogenization and porous media. Springer-Verlag, New York.

Krabbenhoft, K.; Damkilde, L. 2004. Double porosity models for the description of water infiltration in wood. Wood Sci. Technol. 38: 641-659.

Ludwig, R.; Gerke, H.H.; Wendroth, O. 1999. Describing water flow in macroporous field soils using the modified macro model. Journal of Hydrology 215: 135-152.

Perré, P. 1997. Image Analysis, Homogenization, Numerical Simulation and Experiment as Complementary Tools to Enlighten the Relationship between Wood Anatomy and Drying Behavior. Drying Technology Journal 15: 2211 -2238.

Perré, P. 2005. MeshPore : a software able to apply image-based meshing techniques to anisotropic and heterogeneous porous media. Drying Technology Journal 23: 1993-2006.

Perré, P. 2007. Multiscale aspects of heat and mass transfer during drying. Transport in Porous Media 66: 59-76.

Perré, P.; Turner, I. W. 1999a. TransPore: A Generic Heat and Mass Transfer Computational Model for Understanding and Visualising The Drying Of Porous Media. Drying Technology Journal 17: 1273-1289.

Perré, P.; Turner, I. W. 1999b. A 3D version of TransPore : A Comprehensive Heat and Mass Transfer Computational Model for Simulating the Drying of Porous Media. Int. J. Heat Mass Transfer 42: 4501-4521.

Perré, P.; Turner, I.W. 2001a. Determination of the material property variations across the growth ring of softwood for use in a heterogeneous drying model, Part I - Capillary pressure, tracheid model and absolute permeability. Holzforschung 55: 318-323.

Perré, P.; Turner, I.W. 2001b. Determination of the Material Property Variations across the Growth Ring of Softwood for Use in a Heterogeneous Drying Model, Part II - Use of homogenisation to predict bound liquid diffusivity and thermal conductivity. Holzforschung 55: 417-425.

Perré, P.; Turner, I. W. 2002. A Heterogeneous Wood Drying Computational Model that accounts for Material Property Variation across Growth Rings. Chemical Engineering Journal 86: 117-131.

Perré, P.; Turner, I. W.; Remond, R. 2007. Comprehensive Drying Models based on VolumeAveraging: Background, Application and Perspective. Book Chapter in Modern Drying Technology: Vol 1: Computational Tools at Different Scales. Editors E. Tsotsas and A. Mujumdar, Wiley.

Ray, C.; Ellsworth, T.; Valocchi, A.; Boast, C. 1997. An improved dual porosity model for chemical transport in macroporous soils. Journal of Hydrology 19: 270-292.

Showalter, R.E. 1993. Distributed microstructure models of porous media. Int. Series of Numerical Mathematics 114: 155-163.

Siau, J.F. 1995. Wood : in uence of moisture on physical properties. Virginia Polytechnic Institute 
and State University.

Stamm, A.J. 1959. Bound-water diffusion into wood in the fiber direction. For. Prod. J. 9: 27-32.

Wiberg, P.; Morén, T. 1999. Moisture flux determination in wood during drying above fibre saturation point using CT-scanning and digital image processing. Holz als Roh- und Werkstoff. 57: 137-144.

Whitaker, S. 1977. Simultaneous heat, mass, and momentum transfer in porous media: A theory of drying. Advances in Heat Transfer 13: 119-203.

Whitaker, S. 1998. Coupled Transport in Multiphase Systems: A Theory of Drying. Advances in Heat Transfer 31: 1-104.

\section{Nomenclature}

\begin{tabular}{|c|c|c|}
\hline$\overline{\mathbf{D}}$ & Diffusivity tensor & $\mathrm{m}^{2} \mathrm{~s}^{-1}$ \\
\hline$\sigma$ & gravitational constant & $\mathrm{m} \mathrm{s}^{-2}$ \\
\hline$h$ & Intrinsic averaged enthalpy & $\mathrm{J} \mathrm{kg}^{-1}$ \\
\hline $\bar{h}_{b}$ & Averaged enthalpy of bound lic & quid $\mathrm{J} \mathrm{kg}^{-1}$ \\
\hline$\Delta h_{w}$ & Differential heat of sorption & $\mathrm{J} \mathrm{kg}^{-1}$ \\
\hline$\overline{\overline{\mathbf{K}}}$ & Kinetic transport tensor & \\
\hline$\overline{\overline{\mathbf{K}}}_{e f f}$ & Effective thermal conductivity & $\mathrm{W} \mathrm{m} \mathrm{m}^{-1} \mathrm{~K}^{-1}$ \\
\hline$\overline{\overline{\mathbf{K}}}$ & Relative permeability & \\
\hline$\overline{\bar{k}}$ & Absolute permeability & $\mathrm{m}^{2}$ \\
\hline$k_{\mathrm{m}}$ & Mass transfer coefficient & $\mathrm{m} \mathrm{s}^{-1}$ \\
\hline M & Molar mass & $\mathrm{kg} \mathrm{mol}^{-1}$ \\
\hline $\mathrm{P}$ & Pressure & $\mathrm{Pa}$ \\
\hline $\mathrm{q}$ & External heat transfer coefficie & ent $\quad \mathrm{W} \cdot \mathrm{m}^{-2} \cdot{ }^{\circ} \mathrm{C}^{-1}$ \\
\hline $\mathrm{R}$ & Gas constant & $\mathrm{J} \mathrm{mol}^{-1} \mathrm{~K}^{-1}$ \\
\hline $\mathrm{S}$ & Volume saturation & \\
\hline $\mathrm{T}$ & Temperature & K \\
\hline $\mathrm{t}$ & Time & $\mathrm{s}$ \\
\hline V & Velocity & $\mathrm{m} \mathrm{s}^{-1}$ \\
\hline$X$ & Moisture content & \\
\hline$x$ & Molar fraction & \\
\hline
\end{tabular}




\section{Greek Symbols}

$\begin{array}{lll}\chi & \text { Depth scalar } & \mathrm{m} \\ \phi & \text { Porosity } & \mathrm{m}^{3} \mathrm{~m}^{-3} \\ \varphi & \text { Phase potential } & \\ \Phi & \text { Volumetric source term } & \mathrm{W} \mathrm{m} \\ \mu & \text { Dynamic viscosity } & \mathrm{kg} \mathrm{m}^{-1} \mathrm{~s}^{-1} \\ \rho & \text { Intrinsic averaged density } & \mathrm{kg} \mathrm{m}^{-3} \\ \sigma & \text { Surface tension } & \mathrm{N} \mathrm{m}^{-1} \\ \varepsilon & \text { volume fraction } & \\ \omega & \text { Mass fraction } & \end{array}$

\section{Superscripts and Subscripts}

$\begin{array}{ll}\text { a } & \text { Air } \\ \text { b } & \text { Bound } \\ \text { c } & \text { Capillary } \\ \text { eff } & \text { Effective property } \\ \text { fw } & \text { free water } \\ \text { fsp } & \text { fibre satured point } \\ \text { g } & \text { Gas phase } \\ \text { p } & \text { Pressure } \\ \text { s } & \text { Solid phase } \\ \text { sat } & \text { Satured state } \\ \text { T } & \text { Temperature } \\ \text { v } & \text { Vapour } \\ \text { w } & \text { Liquid phase } \\ \infty & \text { Atmospheric }\end{array}$

